\title{
AKOKOID COMPARATIVE WORDLIST
}

\author{
Fádorò̀, Jacob Olúdáre \\ Department of Linguistics and African Languages, \\ University of Ibadan, Ibadan
}

\begin{abstract}
Akokoid, in this paper, refers to the nine speech forms which are spoken in Akoko North-West Local Government Area of Ondo State in South-Western Nigeria. These speech forms are Arigidi, Erushu, Afa, Oge, Aje, Udo, Oyin, Igashi and Uro. Since the 1970's, scholars have lumped these speech forms together as dialects of the same language without any detailed lexicostatistic investigation. Thus, the major objective of this paper is to determine whether the speech forms are really dialects of the same language through lexicostatistic analysis. Data were collected from 34 informants spread across the nine geographical areas where the speech forms are spoken through the direct interview method. In analyzing these data, Swadesh's principles of lexicostatistics were used. The lexicostatistic figures reveal that Arigidi and Erushu are $88.5 \%$ cognate, so they are classified as dialects of Arigidi. Afa, Oge, Aje, Udo, Oyin, Igashi and Uro are $81 \%$ cognate, so they are classified as dialects of the same language called O wọn (meaning 'tongue'). Two distinct but fairly related languages were identified within the nine speech forms. These are Arigidi and Owọn, jointly referred to as Akokoid by virtue of their Akoko root. Therefore, there is no justification for lumping them together as dialects of a single language.
\end{abstract}

\section{Council for Innovative Research}

Peer Review Research Publishing System

Journal: Journal of Advances in Linguistics

Vol 3, No. 1
editor@cirworld.com
www.cirworld.com, member.cirworld.com 


\section{INTRODUCTION}

The Akokoid speech forms have attracted different names from scholars since the 1970s. For example, Hoffman (1974) refers to them as 'Northern Akoko Cluster'; Akinkugbe (1978) refers to them as 'Akokoid'; Capo (1989) calls them 'Amgbe'; Akinyemi (2002) gives them the title 'Arigidi-Amgbe'; while Fadọo (2010) retains 'Akokoid'.

These nine speech forms are all spoken in Akoko North-West Local Government, Ondo State, Nigeria, with the local government headquarters in Oke-Agbe. The speech forms are Arigidi, Erushu, Afa, Udo, Oge, Aje, Oyin, Igashi and Uro. The names given to the speech forms coincide with the names of the communities in which they are spoken. Afa, Udo, Oge and Aje are spoken in Oke-Agbe. See Fig 1, 2 and 3 below for maps.

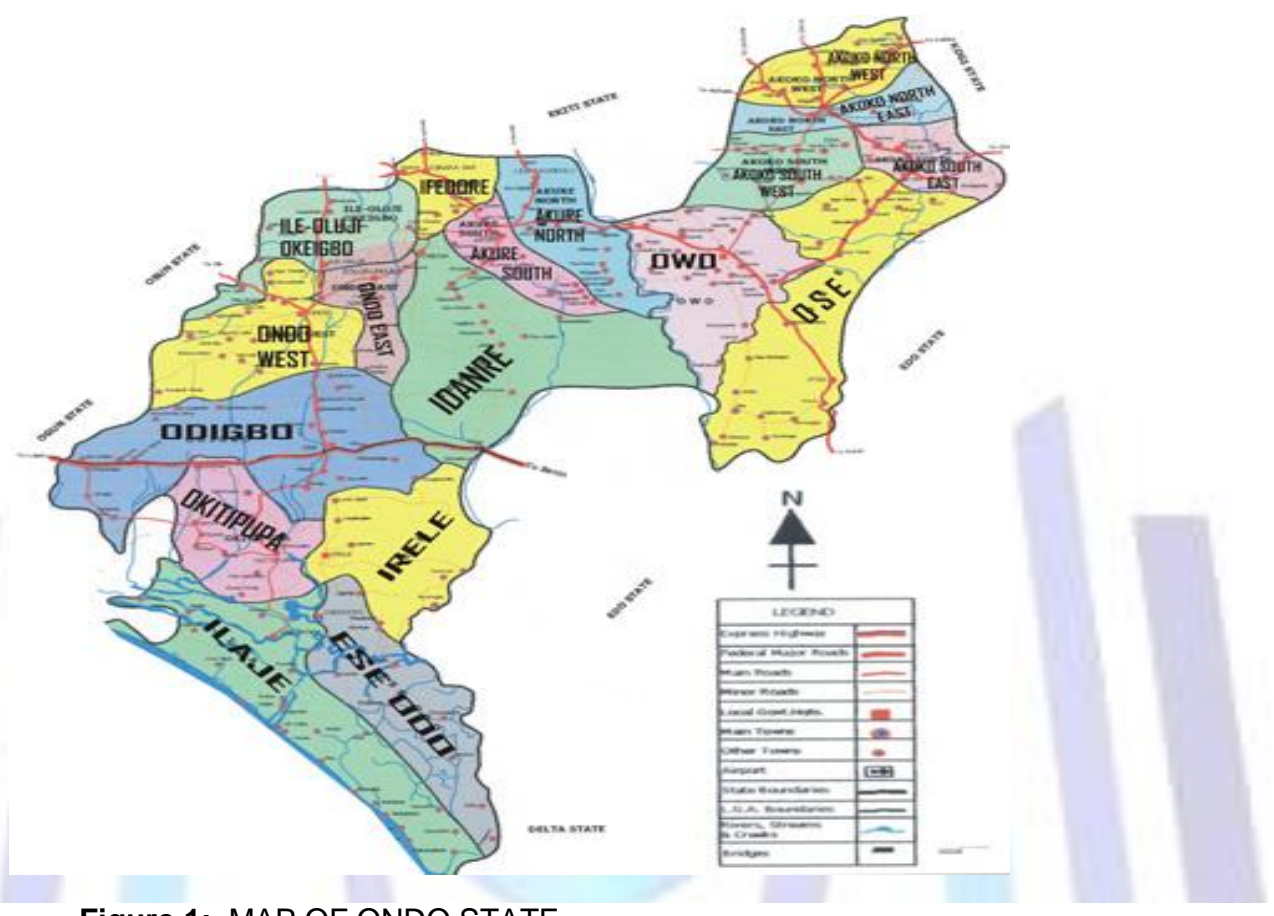

Figure 1: MAP OF ONDO STATE

Source: Akoko North West Local Goernment Council, Ondo State, Nigeria

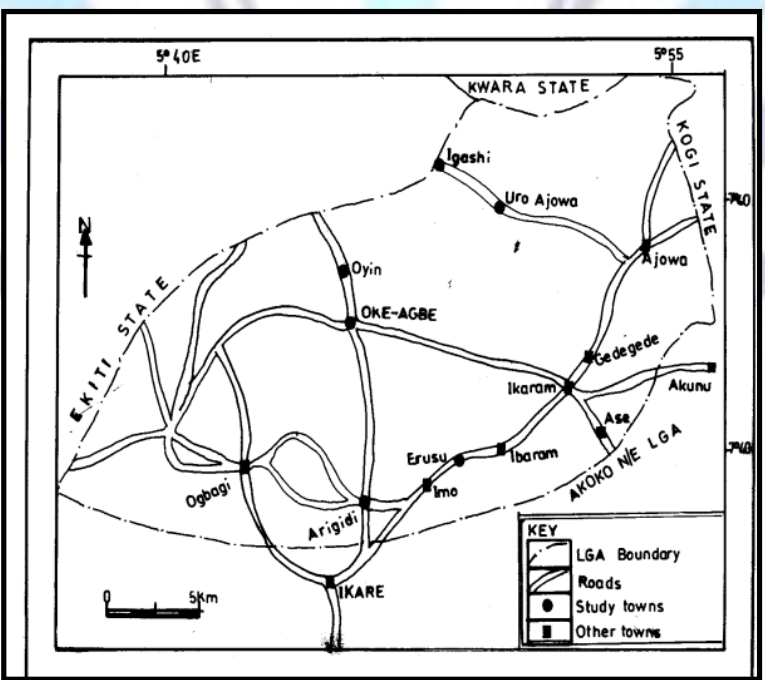

Figure 2: MAP OF AKOKO NORTH WEST LOCAL GOVERNMENT AREA OF ONDO STATE SHOWING THE STUDY TOWNS

Source: Akoko North West Local Goernment Council, Ondo State, Nigeria 


\section{RESEARCH PROBLEM}

The nine speech forms explored in this work have been lumped together by scholars in the 1970s and 1980s without a detailed lexicostatistic investigation. This resulted in the hasty conclusion that they are dialects of the same language. However, a careful look at these speech forms reveals that they cannot be lumped together as dialects of the same language. In fact, speakers in their respective communities do not agree that they speak the same language with speakers in adjacent communities. This calls for a lexicostatistic investigation, which this paper has attempted.

\subsection{OBJECTIVES OF THIS PAPER}

The major goal of this work was determining the status of the Akokoid speech forms. It examined whether the nine speech forms are dialects of the same language or not. It was investigated the level of intelligibility among the over 250,000 speakers who claim that they speak different languages.

\subsection{METHODOLOGY}

The Ibadan 400 wordlist was used to obtain data from 34 informants: Aje (5), Arigidi (5), Oyin (5), Igashi (4), Erushu (3), Afa (3), Oge (3), Udo (3) and Uro (3). Thirty-two of them were non-mobile as well as rural. Twenty-five of them were old, with their ages ranging from 60-80 years old. Twenty-seven of them were males. Thus, the acronym $\operatorname{NORM}(s)$ (which stands for Non-mobile, Old, Rural, Males) was used with little modifications. These informants were contacted and interviewed in their villages.

\subsection{THEORETICAL PERSPECTIVE}

In analyzing the data presented in this work, Pike's (1947) discovery procedure in phonological analysis was employed in determining the phonemic status of the sound systems of Akokoid (Consonants, Vowels and Tones). Secondly, Swadesh's (1951) principles of lexicostatistics were used in classifying the speech forms into two main languages - Arigidi and Ọwọ̀n. Arigidi is made up of Arigidi and Erushu speech forms. Òwọ̀n is made up of Afa, Aje, Udo, Oyin, Igashi, Aje and Oge.

\section{THE CONCEPT OF VARIATION}

Bright (1966:2) makes the following observation:

Within any recognizable speech community, variations are normally found on all levels of linguistic structure - Phonological, grammatical and lexical. Some of the variations are correlated with geographical location... some ... may depend on the identity of the person spoken to or spoken about... other variations are correlated with the identity of the speaker. These include cases of difference between men's and women's speech... linguistic variation may also be correlated with the social status of the speakers (or) with other facts in the social and cultural context.

The excerpt above suggests that variation in language could be determined by different characteristic features. The correlates of linguistic variation could be any of the following:

(i) Geographical location of the speaker (where the people live)

(i) The interlocutors (age disparity and class)

(iii) The topic of discussion or context (what the interlocutors discuss)

(iv) The sex of the speaker/addressee (whether they are males or females)

Bright's comments above also serve as a springboard for this study. The factors spelled out by Bright manifest in the use of language in Akoko. For instance, Dada (2006) and Oyetade (2007) note that there was a significant difference between the different age groups in their ability in Akoko languages and between different occupations. According to them, ability in Akoko languages is gradually dwindling; generally children are not as proficient in Akoko languages as adults. Apart from this, the males have a slightly higher proficiency in their Akoko languages than their female counterparts. Deliberate efforts were made to confirm these observations. This was why we modified NORM(s) in the selection of informants. For example, five of our informants were female, seven were young and six were mobile.

\subsection{FACTORS THAT CAUSE VARIATION IN LANGUAGE}

Many reasons have been ascribed to variation in language. One of the prominent characteristics of language in general is the fact that language is dynamic, that is, it is not monolithic. It changes from time to time. Brook (1973:162) remarks that 'the spoken language is like a living person growing older continuously but so slowly that it is hard to point to any one changing feature'.

One of the factors responsible for variation in language is the social organization of the speech community itself. This includes differences in age, sex, social status and the setting in which interaction takes place. Bright and Ramaanjan (1964) capture this in the following words: 
"What is termed the social dimension of linguistic variation is correlated with the socially established identity of the speaker/or the person addressed or mentioned."

Furthermore, regional varieties of language develop as different norms arise in the usage of groups who are separated by some kind of geographic boundary. Geographical distance is an important factor that causes variation in language. Fromkin and Rodman (1993) assert that regional diversity develops when people are separated from each other geographically and socially. This is because the changes that occur in the form spoken in one area or group do not necessarily spread to another. Dialect differences tend to increase proportionately to the degree of communicative isolation between the groups. Communicative isolation refers to a situation such as existed between America, Australia, and England in the eighteenth century. Other factors responsible for variation in speech are sex, time, and education. The data presented in this paper highlights the similarities and differences attested in Akokoid.

\section{COMPARATIVE WORDLIST}

In an attempt to expose the internal relationship within the Akokoid speech forms, two hundred lexical items consisting of one hundred nouns (such as names of body parts and natural phenomena, like sun, moon, star, fire, rain, river, day, night etc and one hundred simple verbs (like go, come, run, eat, see, die, etc.) are presented here. Because of their everyday significance, these words are believed to be least prone to change or borrowing, as every culture and linguistic group has words for them. These items are presented with their Yorùbá and English equivalents in Table 1 below:

TABLE 1: COMPARATIVE WORDLIST

\begin{tabular}{|c|c|c|c|c|c|c|c|c|c|c|}
\hline Gloss & Arigidi & Erushu & Afa & Udo & Oyin & Igashi & Oge & Uro & Aje & Yorùbá \\
\hline Head & Egíri & egírí & Igíri & igíri & igíri & igíri & igíri & egírí & igíri & orí \\
\hline Hair & i Sírí & iSírí & ìtìri & iś̂́î́i & ítíri & ifîrí & iś̃î́î́ & iśîrí & iś̂́̌ & irũ \\
\hline Eye & ódzù & ódzù & Ídzù & íd3ù & ídzù & ídzù & ídzù & ídzù & ídzù & odzú \\
\hline Ear & Oto & oto & Útó & útó & útó & uto & útó & útó & útó & etí \\
\hline Nose & od3uw̄ं & úw̃ & ùพ์ & úw̃̃์ & ùพ์ & úw̃ố & úw̃ố & úw̃ố & úw̃ố & imû́ \\
\hline Mouth & õrũ & òrũ & $\begin{array}{l}\text { Odò } \\
\text { ru }\end{array}$ & òrũ & òrũ & odòrũ & òrũ & odòru & òrũ & عnũ \\
\hline Tooth & éjĩĩ & éñi & éfĩ & éfĩi & épĩ & épī & épī & épī & éj̄i & ejí \\
\hline Tongue & ع́r $\varepsilon$ & íre & ér̃ $\tilde{r} \tilde{\varepsilon}$ & 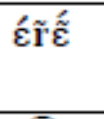 & $e^{\prime} \tilde{r} \tilde{r} \tilde{\varepsilon}$ & iٓ̃ $\tilde{\varepsilon}$ & 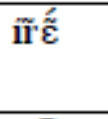 & 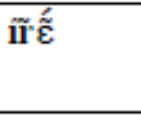 & î̃̃ & $\begin{array}{l}\text { aw̃ố/a } \\
\text { กõ์ }\end{array}$ \\
\hline Chin & àgbõ & àîngbà & $\begin{array}{l}\text { àjịng } \\
\text { bà }\end{array}$ & $\begin{array}{l}\text { àjÎm } \\
\text { gbà }\end{array}$ & $\begin{array}{l}\text { àymgb } \\
\text { à }\end{array}$ & $\begin{array}{l}\text { àymgb } \\
\text { à }\end{array}$ & $\begin{array}{l}\text { àying } \\
\text { bà }\end{array}$ & $\begin{array}{l}\text { àgbaymm } \\
\text { gbà }\end{array}$ & àŷmgbà & àgbō \\
\hline Beard & isierele & i $\int$ il $\varepsilon$ & ìsile $\varepsilon$ & il $\varepsilon$ & ile & ùlè & ùlદ̀ & ulદ̀ & eŝ́î́í ilè & irūgbō \\
\hline Neck & úgo & úgo & ògúgo & ogúgo & ògúgo & ut $\int \varepsilon ́$ & ùt $\int \varepsilon ે$ & ùt $\int \varepsilon ે$ & ut $\int \varepsilon$ & orū \\
\hline $\begin{array}{l}\text { Breast } \\
\text { (female) }\end{array}$ & عpõ & Épo & ípo & ípo & ípo & ípò & ípò & ípò & ípò & omú \\
\hline Heart & okã & okã & okã & okã & okã & okã & okã & okã & okã & okã \\
\hline $\begin{array}{l}\text { Belly } \\
\text { (external) }\end{array}$ & ogo & ogo & ìgo & ìgo & ìgo & ìgo & ìgo & ìgo & ígó & ikū \\
\hline Navel & عkpõ & عkpõ & Íkó & ípố & igo & ipo & ikpố & ípṍ & ípṍ & idodo \\
\hline Back & os & osũ & òs & òsũ & òsũ & òsũ & òsũ & òsũ & òsũ & غेรีi \\
\hline Hand & ówo & ówó & úwó & úwó & úwó & uwo & úwó & úwó & úwó & owó \\
\hline $\begin{array}{l}\text { Nail } \\
\text { (finger or } \\
\text { toe) }\end{array}$ & $\varepsilon \mathbf{k} \tilde{\varepsilon}$ & akík $\tilde{\varepsilon}$ & $\begin{array}{l}\text { ika } \\
\text { m } \tilde{\varepsilon}\end{array}$ & ìnyà & ìnyà & ìnyà & ínyà & ínyà & ínyà & èékấnấ \\
\hline Buttocks & 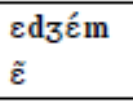 & ihàjẽ & igo & ibõ & íbõ & inō & inō & inō & (દેt $\left.\int \grave{E}\right)$ & ìdí \\
\hline Penis & oko & oko & İlù & índú & índú & índú & ìnū & ìndù & ìndù & okó \\
\hline Thigh & bàlàntà & ilpa & $\begin{array}{l}\text { Bàlàt } \\
\text { ùhò }\end{array}$ & $\begin{array}{l}\text { bàlàt } \\
\text { ùo }\end{array}$ & if & (okoko) & bàlàtì & (òkókó) & bàlàtùò & itā \\
\hline Leg & ùho & ùho & Ühò & ùhò & uhò & ùhò & ùhò & ùhò & ùhò & $\varepsilon s \varepsilon े$ \\
\hline Vagina & $\varepsilon$ กิ๊ & (órolūi) & (oníi) & î́ấ & î́ấ & i็ấ & iกấ & iֹấ & iกล์ & òbò \\
\hline Body & edze & èdze & idz3i & idzi & idzi & idzi & idzi & igèrèd3í & omidzio & ara \\
\hline
\end{tabular}




\begin{tabular}{|c|c|c|c|c|c|c|c|c|c|c|}
\hline Skin & (awo) & alà & àlà & alà & alà & alà & alà & alà & alà & awo \\
\hline Bone & ékpe & íkpi & ikpí & ikpĩ & íkp $\tilde{\varepsilon}$ & ikpi & ikpĩ & ikpi & ékpi & egũgũ \\
\hline Blood & $\bar{\varepsilon} \mathrm{d} 3 \bar{\varepsilon}$ & $\bar{\varepsilon} \mathrm{d}_{3} \bar{\varepsilon}$ & $\bar{\varepsilon} \mathrm{d} 3 \bar{\varepsilon}$ & $\bar{\varepsilon} \mathrm{d} 3 \bar{\varepsilon}$ & $\bar{\varepsilon} \mathrm{d} 3 \bar{\varepsilon}$ & غेd3દ̀ & غेd3દ̀ & غेd3 & غेd3દ̀ & غेd3 غे \\
\hline Saliva & it $\tilde{\varepsilon}$ & it $\tilde{\varepsilon}$ & út $\tilde{\varepsilon}$ & út $\tilde{\varepsilon}$ & út $\tilde{\varepsilon}$ & it $\varepsilon$ & út $\bar{\varepsilon}$ & 宅t & it & itó \\
\hline Urine & ùto & utó & ìtó & ìtó & itó & ìtó & ìtó & ìtó & ìtó & ìtò \\
\hline Faeces & $\varepsilon \mathrm{m} \varepsilon$ & $\varepsilon \mathrm{m} \varepsilon$ & ímí & ímí & ímĩ & íní & ímí & ímí & ímí & imííigbé \\
\hline Water & edzî & èdzi & ùdzi & údzi & ùdzi & ùdzi & ùdzi & ùdzi & ùdzi & omi \\
\hline Soup & aj $\varepsilon$ & àjह & àj $\varepsilon$ & àjह & àjह & àj $\varepsilon$ & àj $\varepsilon$ & àj $\varepsilon$ & àj $\varepsilon$ & ob غे \\
\hline Meat & ar̃̃ã & àr̃̃ã & àr̃̃ã & àr̂̃ã & àr̃̃̃a & àr̃̃̃a & àr̃̃ã & àr̂̃ã & àr̃̃ã & 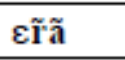 \\
\hline Fat & òrà & òrárã & ùh हे & úh $\varepsilon$ & úh $\varepsilon$ & úh $\varepsilon$ & úh $\varepsilon$ & ùh غे & òrá & úh $\varepsilon$ \\
\hline Fish & eso & iso & $i \phi \varepsilon$ & $i \oint \dot{\varepsilon}$ & $i \oint \dot{\varepsilon}$ & 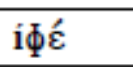 & $i \phi \varepsilon$ & $i \phi \varepsilon$ & i $\phi \dot{\varepsilon}$ & $\varepsilon d 3 a$ \\
\hline Oil & ógo & úgo & úgo & úgo & úgo & úgo & úgo & úgo & úgo & ekpo \\
\hline Salt & (owò) & ut & ut Ŝ́ & út $\int \tilde{\varepsilon}$ & út $\int \tilde{\varepsilon}$ & it $\int a$ & ut śsi & ut Sí & ut Śi & ijò \\
\hline Wine & orà & orà & ùrà & ùrà & ùrà & ùrà & ùrà & ùrà & ùrà & otí \\
\hline Palm wine & umū & umū & imū & imū & ímū & imû́ & imù & ímũ & imū & $\begin{array}{l}\text { Emũ/ò } \\
\text { gùrò }\end{array}$ \\
\hline Yam & i $\int \tilde{\varepsilon}$ & i $\int \tilde{\varepsilon}$ & àd3u & ad3u & àd3u & ìti & ìsi & ìsi & ìsi & isu \\
\hline Cassava & ógòrò̀lò & Ogòrò̀lò & ogorolo & ogorolo & ógòròlò & ógòròlò & ógòròlò & 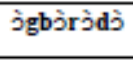 & ògòròlò & غेgर́ \\
\hline $\begin{array}{l}\text { Guinea } \\
\text { corn }\end{array}$ & it $\int \bar{\varepsilon}$ & it $\int \grave{\varepsilon}$ & ìt & it غे & it غे & ìtรे & ìšे & ìs हे & ìšे & $\begin{array}{l}\text { okà } \\
\text { bàbà }\end{array}$ \\
\hline Maize & $\begin{array}{l}\text { igbàdo } \\
\end{array}$ & igbàdo & igbàdo & igbàdo & igbàdo & igbàdo & àgbàdo & iggbàdo & igbàdo & àgbàdo \\
\hline Beans & (Èrદ̀hè) & èdzì & èdzì & èdzì & èdzì & èdzì & èdż̀̀ & èdzì & èdzì & ह̀wà \\
\hline Pepper & $\varepsilon \int \tilde{\varepsilon}$ & $\varepsilon \int \tilde{\varepsilon}$ & $\varepsilon ́ t \varepsilon$ & 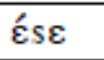 & ع́t & દtغેjò & ع́s $\varepsilon$ & Esદેjò & 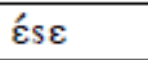 & ata \\
\hline Okra & óhũ & óhũ & ínru & ínru & ínru & ínru & ínru & ínru & ínru & Ilá \\
\hline Orange & òrom̀bó & òromìbó & $\begin{array}{l}\text { òrom̀ } \\
\text { bó }\end{array}$ & $\begin{array}{l}\text { òrom̀ } \\
\text { bó }\end{array}$ & ì̀òmú & ilèmú & òromibó & isō & ilòmí & $\begin{array}{l}\text { òroǹ̀bó } \\
\text { /osā }\end{array}$ \\
\hline Groundnut & Èkpà & Èkpà & ह̀ kppà & ह̀kpà & ड̀kparado & Èlpàràdó & हे̀pà & Èkpàràdó & Èkpà & Èkpà \\
\hline Kolanut & e Só & etò & $i \int \hat{i}$ & it $\int i$ & it $\int \hat{e}$ & it $\int \mathrm{è}$ & it $\int \grave{e}$ & it Sè & it $\int \dot{e}$ & obì \\
\hline Tobacco & tábà & tábà & tábà & itábà & tábà & itábà & tàbà & ìtabà & tábà & tábà \\
\hline Cotton & orúru & orúru & orúru & orúru & orúru & ètituòwú & orúrú & òwú & orúru & òwú \\
\hline Oil palm & $\begin{array}{l}\text { etSitS } \\
\text { oògò }\end{array}$ & úgo & - & $\begin{array}{l}\text { itsts } \\
\text { ógo }\end{array}$ & $\begin{array}{l}\text { it ftot } \\
\text { Sógo }\end{array}$ & $\begin{array}{l}\text { itfóts } \\
\text { ougù }\end{array}$ & $\begin{array}{l}\text { ít SotS } \\
\text { Gúgo }\end{array}$ & ógò & $\begin{array}{l}\text { it SotSo } \\
\text { ùgò }\end{array}$ & $\begin{array}{l}\text { ekpok } \\
\text { pukpa }\end{array}$ \\
\hline Seed & a se & èso & kóro & & àti & ógò & àsi & àsi & àsi & èso \\
\hline Grass & è $\int i \int i$ & isísi & isisi & 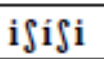 & $i \int i \int i$ & Sí̧ìrì & SiSirì & Sisirs & ìjî̀ìì & koríko \\
\hline Tree & óhõ & óhõ & üyro & únro & ú́nro & ónro & ónro & ónro & ứgro & igi \\
\hline Leaf & $\operatorname{im} \bar{\varepsilon}$ & ìm $\bar{\varepsilon}$ & $\varepsilon \mathrm{m} \bar{\varepsilon}$ & $\varepsilon^{\prime} \mathrm{m} \bar{\varepsilon}$ & $\varepsilon^{\prime} \mathbf{m} \bar{\varepsilon}$ & 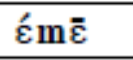 & ع́m $\tilde{\varepsilon}$ & 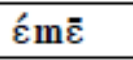 & $\varepsilon^{\prime} \mathbf{m} \bar{\varepsilon}$ & ewé \\
\hline Thorn & غेd3 $\tilde{\varepsilon}$ & is & ud3 $\varepsilon$ & ud3k & ud3र́ & $\varepsilon \mathrm{d} 3 \hat{\varepsilon}$ & $\varepsilon d 3 \hat{\varepsilon}$ & غ̀d3र́ & ud3र́ & غ̀gû́ \\
\hline Charcoal & id $\overline{31}$ & 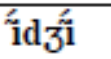 & és̄ì & ésī & ési & és̄i & ésì & ési & éśí & èédú \\
\hline Smoke & újù & úwú & ówú & ówú & ówú & úvú & ówú & úwú & ówú & èéfí \\
\hline Fire & et So & kit So & ító & ító & ító & itso & isó & ísó & isó & inấ \\
\hline Ashes & odõ & ódố & ónố & ónó & óndó & ondo & ónó & óndó & ứdó & èérú \\
\hline
\end{tabular}




\begin{tabular}{|c|c|c|c|c|c|c|c|c|c|c|}
\hline Pot & àt & àt Sà & àt $\int a ̀ ~$ & 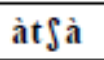 & àt $\int a ̀ ~$ & 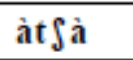 & àt & àt 〔à & 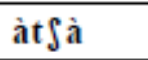 & ikòkò \\
\hline Calabash & ekû́ & ekứ & ikứ & ekṹ & ikû́ & ikú & ikû́ & ìfò & ikû́ & igbá \\
\hline Mortar & ebú & ebú & ibú & ibú & ibú & ígú & igú & igú & íbú & odó \\
\hline Knife & is & ù $\int \tilde{\varepsilon}$ & $\mathbf{u} \Phi \tilde{\varepsilon}$ & $\mathbf{u} \phi \tilde{\varepsilon}$ & $\mathbf{u} \Phi \tilde{\varepsilon}$ & $\mathbf{u} \Phi \tilde{\varepsilon}$ & $\mathbf{u} \Phi \tilde{\varepsilon}$ & ú $\phi \tilde{\varepsilon}$ & ú $\phi \tilde{\varepsilon}$ & òb $\varepsilon$ \\
\hline Hoe & úhõ & uhō & grà & prà & grà & grà & grà & grà & grà & okó \\
\hline Axe & ذ̀น & غ̀ngغ̀ & ogró & ogró & onró & غे̀g & غेng غે & غે̀g & غे̀g غે & àáké \\
\hline Machete & Ér $\varepsilon$ & íre & àgb $\varepsilon$ & àgbé & àgbé & àda & àdá & àdá & àdá & àdá \\
\hline Spear & òkò & òkò & òkò & òkò & ùkò & ùkò & (èt $\left.\int i\right)$ & ̀ेkò & òkò & òkò \\
\hline Horn & غેhõ & غेhõ & ihó & ìnró & ìnró & ògogo & ìnró & ùnımgbà & inmwá & ìwo \\
\hline Iron & írũ & irĩ & úr $\tilde{\varepsilon}$ & úr $\tilde{\varepsilon}$ & úr $\tilde{\varepsilon}$ & irĩ & írĩ & úr $\tilde{\varepsilon}$ & úr $\tilde{\varepsilon}$ & irĩ \\
\hline Mat & ìsà & at & $\begin{array}{l}\text { (akòr } \\
\text { ógbá) }\end{array}$ & às $\tilde{\varepsilon}$ & $a \int \varepsilon$ & orò $\int \varepsilon$ & ì̀ $\int \varepsilon$ & ì̀ $\int \varepsilon$ & ì̀ $\int \varepsilon$ & Ení \\
\hline Basket & ehō & îhõ & ínrá & (ikúrùu) & ínrá & ínrá & ínrá & ínrá & ínrá & $\begin{array}{l}\text { agbõ/a } \\
\text { kpغ̀pغे }\end{array}$ \\
\hline Bag & àkpò & àkpò & àkpò & àkpò & àkpò & àkpò & àkpò & àkpò & àkpò & àkpò \\
\hline Rope & òkū & òkū & òkū & òkū & òkū & uka & òkū & òkū & okū & okū \\
\hline Needle & ikéné & ìné & ùné & ùné & ùné & ùken $\varepsilon$ & ùné & ùn & ùné & abśré \\
\hline Thread & orúru & orúru & orúru & orúru & orúru & òwú & orúru & òwú & orúru & òwú \\
\hline Hat & éwà & iva & idú & idú & idú & ídú & idú & idú & idú & filà \\
\hline Shoe & bàtà & bàtà & bàtà & bàtà & bàtà & ibàtà & bàtà & ìhàūs & bàtà & bàtà \\
\hline Money & òkùbà & òkùbà & egó & ewó & ewó & evo & evo & evó & ewó & owó \\
\hline Town & ègú & egú & ikú & ibú & gú & ìgú & ìgú & ìgú & ihú & ìlú \\
\hline Well & kānga & kòga & kล̄inga & kānga & kānga & ikànga & kōnga & kānga & kōnga & kāga \\
\hline Market & adza & adza & adzá & ádzá & ádzá & adza & ádzá & ádzá & ád3á & od3à \\
\hline Farm & ìjà & ùwà & ùwà & ùwà & ùwà & ùwà & ùwà & ùwà & ùwà & okó \\
\hline Sea & òkũ & òkũ & òkũ & òkũ & òkũ & òkũ & (ihà) & òkũ & òkũ & òkũ \\
\hline Stone & éta & íta & íta & íta & ita & íta & íta & íta & íta & òkúta \\
\hline Mountain & èdè & ídè & ídì & ídì & ídi & ìgìdì & ìdí & (ùgbà) & ídì & òkè \\
\hline Sand & $\eta \bar{\varepsilon} \eta \bar{\varepsilon}$ & ijẽ $\tilde{\varepsilon} \bar{\varepsilon}$ & ле̄̄n $\bar{\varepsilon}$ & 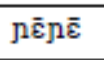 & 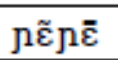 & กล̃ก' & $\mathrm{n} \bar{\varepsilon} \mathrm{\rho} \bar{\varepsilon}$ & $\mathrm{n} \bar{\varepsilon} \mathrm{\rho} \bar{\varepsilon}$ & กล̃กี & กล̃กי̄ \\
\hline Cow & àrogò & àrogò & àràgò & arāgò & àragò & imàlu & àràgò & àràgò & màlúù & màlúù \\
\hline Sheep & adõ & ándá & ayá & ángá & aygá & ándá & áná & ándá & ándá & àgû́tā \\
\hline Dog & ofo & ófó & ópú & ópú & ópú & ópú & ópú & ópú & ópú & adzá \\
\hline Rat & odzúwwà & iff $\tilde{\varepsilon}$ & òdírì & òdí & òdí & òdí & òdí & òdí & òdí & eku \\
\hline Monkey & obo & obo & akấ & áká & àtò & amèǹtย & áká & áká & áká & ̀ेbo \\
\hline Ground & es̃i & es̃ & éśí & éśî́ & éŝ́i & é $\mathrm{i}$ & ésín & iş́i & é $\int_{1}^{\prime}$ & ilદ̀ \\
\hline Earth & ifa & ít Sa & it Sa & it Sa & it §á & it $\int a$ & it Sa & it Sa & it Sa & ajé \\
\hline Rain & edżi & èd3i & ud3í & ùd3ii & ùd3i & ùdzii & ùd3ii & ùd3i & ùd3ii & òdzò \\
\hline Sunshine & ùhò & ùhò & ùhò & ùhò & ùhò & ùhò & ùhò & ùho & ùhã & ò̀̀rû́ \\
\hline Moon & eridza & erid3a & $\begin{array}{l}\text { ò ùk } \\
\text { pá }\end{array}$ & $\begin{array}{l}\text { à Sùk } \\
\text { pá }\end{array}$ & ósủlpá & òs ùlpá & et 5 igbà & èsìgbà & ò Sùkpà & ô Sùkpà \\
\hline War & olo & oló & oló & òlo & òlo & òlo & òlo & òlo & òlo & ogũ \\
\hline Song & isji & u $\int \tilde{\varepsilon}$ & ùsĩ & ùŝ̃i & ùs̃i & ùsิî & ùs $\varepsilon$ & ùs $\varepsilon$ & $\overline{\mathbf{u}} \int \tilde{\varepsilon}$ & orĩ \\
\hline
\end{tabular}




\begin{tabular}{|c|c|c|c|c|c|c|c|c|c|c|}
\hline Eat & d30 & d3o & d3u & d3u & d3u & d3u & d3iho & d3u & d3u & d3 $\varepsilon$ \\
\hline Drink & bo & bo & bo & bo & bo & go & go & gó & bo & $\mathbf{m u}$ \\
\hline Swallow & t Síromí & t síròmi & tír̀̀mũ & tirò̀mi & tiròmū & ròmũ & síròmi & úròmĩ & síròmĩ & gbémī \\
\hline Vomit & kpà & kpà & kpà & pà & pà & pà & pà & pà & pà & bì \\
\hline Urinate & tó & tò & to & tó & tó & to & tò & tò & tò & tò \\
\hline Defecate & $\tilde{\mathrm{\jmath}} \tilde{\varepsilon}$ & $\tilde{\mathrm{j}} \tilde{\varepsilon}$ & $\tilde{\mathrm{ji}}$ & jii & 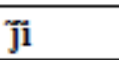 & 元i & $\tilde{\mathrm{j} i}$ & jii & $\tilde{\mathrm{ji}}$ & jàgbé \\
\hline Give birth & tSúwõ & tรúwõ & Súwō & súwō & tรúwร̃ & tSúwō & tร ม́พับิ & t Súw̃ & Súw̃ & bímo \\
\hline Die & kû́ & kû́ & kú & kú & kú & kú & kú & kú & kú & kú \\
\hline Sleep & $\int \tilde{\varepsilon}$ & $\int \tilde{\varepsilon}$ & $\int \tilde{\varepsilon}$ & $\int \tilde{\varepsilon}$ & $\int \tilde{\varepsilon}$ & $\int \tilde{\varepsilon}$ & $\int \varepsilon$ & $\int \tilde{\varepsilon}$ & $\int \tilde{\varepsilon}$ & sū \\
\hline Go & kéwe & vè & vè & mevè & vè & vè & rívè & rívè & vè & lo \\
\hline Come & kéwa & và & và & meva & va & va & và & róvà & va & wá \\
\hline Return & bídzehè & radze & usī & gusì & gusì & lísì & lisì & lisì & lísì & kpadà \\
\hline Fall & tSõ & Sõ & jé & jé & jé & jé & jé & jé & jé & Subú \\
\hline Walk & $\mathrm{d} \overline{3} \overline{1}$ & d3̧غे & $s \bar{\varepsilon}$ & $s \bar{\varepsilon}$ & $s \bar{\varepsilon}$ & $s \bar{\varepsilon}$ & $s \bar{\varepsilon}$ & $\int \bar{\varepsilon}$ & sغे & $\overline{1 \overline{1}}$ \\
\hline Run & tukò & rise & Sí & Si & $\Phi \mathbf{i}$ & $\Phi \mathbf{i}$ & Si & Sí & Sì & sáré \\
\hline Fly & kòhò & hò & hú & hù & régo & hù & hù & hù & hù & fò \\
\hline Jump & tá Síjá & ré & gbálàti & bé & ré & bé & bé & bé & bé & bé \\
\hline See & rí & rí & rí & gò & rí & rí & rí & gò & gò & rí \\
\hline Hear & $\int \varepsilon$ & $t \int \varepsilon t \int_{0}$ & $\int \varepsilon$ & Sótó & $\int \varepsilon^{\prime}$ & $\int \varepsilon$ & $\int \varepsilon$ & $\int \varepsilon$ & $\int \varepsilon$ & gbó \\
\hline Touch & $\begin{array}{l}\text { gbốhố } \\
\text { Só }\end{array}$ & $\begin{array}{l}\text { gbów } \\
\text { óke }\end{array}$ & $\begin{array}{l}\text { fow } \\
\text { óbà }\end{array}$ & $\begin{array}{l}\text { gb6w } \\
6 \text { pà }\end{array}$ & $\begin{array}{l}\text { gbow } \\
\text { ópà }\end{array}$ & bùwoké & buwókā & fow6bà & fowóbà & fow6bà \\
\hline Know & rấ & rấ & rà & rā & rà & rā & rā & rā & rā & mō \\
\hline Remember & jídã & jéǹda & jélà & jéc̀nấ & jèǹdá & jèǹda & jénā & jèǹdá & jedá & rấtí \\
\hline Learn & kó & kó & kó & kó & kó & kó & kó & kó & kó & kó \\
\hline Laugh & wõ & wõ & wõ & wõ & wõ & wõ & wõ & wõ & wõ & réદ̀ríi \\
\hline Sing & Si $i \int \varepsilon$ & kùt $\int \varepsilon$ & kòtS $\bar{\varepsilon}$ & kòsii & kòs $\tilde{\varepsilon}$ & kòs $\tilde{\varepsilon}$ & kòs $\tilde{\varepsilon}$ & kòs $\tilde{\varepsilon}$ & kòs $\tilde{\varepsilon}$ & korĩ \\
\hline Dance & rè & rè & jह̀ & jè & $\mathrm{j} \dot{\varepsilon}$ & jè & $\mathrm{j} \dot{\varepsilon}$ & jø̀ & jह̀ & d3ó \\
\hline Greet & bá & ba & ba & bá & bà & wà & wá & wa & và & kí \\
\hline Send & dố & dố & nố & nó & ndó & dó & nố & dố & nố & rá́ \\
\hline Refuse & gó & gó & gó & gó & gó & gó & gó & gó & gó & kò \\
\hline Take & bà & gbà & gbà & gbà & gbà & gbà & gbà & gbà & gbà & gbà \\
\hline Steal & dè & de & dèd3i & dí & dèdi & dí & dèdi & dèdi & dèdzi & d3í \\
\hline Sell & sa & sa & Sa & Sá & Sa & Sá & $t \int a$ & t $\int \bar{a}$ & $t \int a$ & rà \\
\hline Give & gbàjấ & gbàjã & gbajjấ & gbàjāā & gbàj̃ã & gbàjã & gbàjã & gbàjã & gbàjã & fú́ \\
\hline Buy & bà & dà & bà & dà & dà & dà & dà & dà & dà & rà \\
\hline Pay & sعm $\tilde{\varepsilon}$ & $\operatorname{s\varepsilon m} \bar{\varepsilon}$ & Sewó & Si & Sewó & Sévó & Si & Seó & Sewó & sãwó \\
\hline Count & kà & kà & kà & kà & kà & kà & kà & kà & kà & kà \\
\hline Divide & mố & mõ & mã & má & mấ & mã́ & mấ & mã & mã & kqú \\
\hline Shoot & mí́ & "ె̄bõ & jె̄bõ & jె̄bõ & "ె̄ibõ & jె̄bõ & స̄jbõ & ]̄jbõ & ]̄jbõ & "ె̄bõ \\
\hline Kill & kó & kpo & kpú & kpú & kpu & kpú & kpú & kpú & kpú & kpa \\
\hline Cook & rā & rã & rã & rấ & rẫ & rấ & rā & rã & rã & Sè \\
\hline
\end{tabular}




\begin{tabular}{|c|c|c|c|c|c|c|c|c|c|c|}
\hline Fry & sũ & sũ & sṹ & sû́ & tû́ & $s \varepsilon$ & $\overline{\phi i}$ & ф́́ & ф́í & díi \\
\hline Roast & tSã́ & t $\int a \tilde{a}$ & tứ & tû́ & sû́ & tá & sṹ & tá & sṹ & sû́ \\
\hline Pound & bu & bu & bú & bú & bú & gú & gú & gú & bú & gû́ \\
\hline Grind & ró & ró & ro & ró & ro & ró & ró & ró & ró & lò \\
\hline $\begin{array}{l}\text { Plait } \\
\text { (hair) }\end{array}$ & bá & pá & kpá & pấ & pá & pa & pã & pá & pà & kó \\
\hline Pull & já & ja & ja & já & já & já & já & já & já & fà \\
\hline Break & fó & fó & fó & fó & fó & fó & fó & fó & fó & fó \\
\hline Dig & gb $\varepsilon^{\prime}$ & gbé & gb $\varepsilon^{\prime}$ & gb的 & gb $\mathbf{c}$ & gb $\varepsilon^{\prime}$ & gb $\varepsilon^{\prime}$ & gb $\varepsilon^{\prime}$ & gb $\varepsilon^{\prime}$ & gb́ś \\
\hline Carve & gb $\varepsilon$ & gbé & gbé & gbé & gb $\varepsilon^{\prime}$ & gb $\varepsilon$ & gb $\varepsilon^{\prime}$ & gbé & gb $\varepsilon$ & gb́́ \\
\hline Pierce & gû́ & gû́ & $\mathrm{v} \varepsilon ́$ & gú́ & gû́ & gû́ & kû́ & ja & gárà & gû́ \\
\hline Cover & Đgù & dú & dú & dú & wù & bù & ggù & du & Đgù & dé \\
\hline Close & tú & tú & tì & tì & tì & tì & dú & dú & (wúsi) & tì \\
\hline $\begin{array}{l}\text { Build } \\
\text { (house) }\end{array}$ & kóso & káso & kéфe & kóeథe & húéథe & kóéфe & kóéđe & kóéфe & mấéфe & kólé \\
\hline Split & lá & lá & mõ & lá & lá & lá & mà & ma & la & là \\
\hline Bury & lú & lú & (si) & lú & lú & dzú & dzú & dzú & lu & sีi \\
\hline Dwell & gbé & gbé & gbé & gbé & gbé & gbé & gbé & gbé & gbé & gbé \\
\hline $\begin{array}{l}\text { Hold (in } \\
\text { hand) }\end{array}$ & d3ádà & jadà & dzàda & d3ádà & jádà & gbèhodá & d3ádà & gómàdzi & gbàda & mū \\
\hline Know & rấ & rấ & rà & rā & rà & rā & rā & rā & rā & $\mathrm{m}$ \\
\hline Swell & $(\mathrm{k} \bar{\varepsilon})$ & wù & gú & gú & hú & wù & wù & wù & hu & wú \\
\hline Sweep & tố & tố & $\int \varepsilon$ & $\int \varepsilon^{\prime}$ & $\int \varepsilon^{\prime}$ & (kpà) & t $\int \varepsilon ́$ & (kpá) & d3ź & gbá \\
\hline Sew & $\int \varepsilon$ & $t \int \grave{\varepsilon}$ & hú & hú & hù & rót \at $\int 2$ & $\mathrm{t} \int \grave{\varepsilon}$ & $\int \varepsilon$ & $\int \grave{\varepsilon}$ & rấ \\
\hline $\begin{array}{l}\text { Put on } \\
\text { (cloth) }\end{array}$ & tSé & tSao & tí & gbàti & gbàtí & gbàbòti & $\begin{array}{l}\text { gbàw } \\
\text { ùsi }\end{array}$ & gbàsí & sawù & $\begin{array}{l}\text { woे } \\
\text { (woSo) }\end{array}$ \\
\hline $\begin{array}{l}\text { Beat } \\
\text { (person) }\end{array}$ & $s \varepsilon$ & $s \varepsilon$ & ri & lí & Sō & dàdzi & d3í & d3í & d3í & lù \\
\hline $\begin{array}{l}\text { Beat } \\
\text { (drum) }\end{array}$ & lé & le & li & lí & lí & lí & lí & lí & lí & lù \\
\hline Bite & rád3ò & rád3ò & kédzu & ró́d3u & gúrud3u & péd3u & péd3u & péd3u & $\mathrm{p} \varepsilon \mathrm{d} 3 \mathrm{u}$ & géd3ع \\
\hline Stand (up) & $\int \tilde{1 j} \tilde{\varepsilon}$ & t $\int u ̀ w \bar{\varepsilon}$ & ùw $\tilde{\varepsilon}$ & Suwé & Suw̃ $\tilde{\varepsilon}$ & $t \int \varepsilon_{j i}$ & dốw $\bar{\varepsilon}$ & ùw $\tilde{\varepsilon}$ & sû́w̃ $\bar{\varepsilon}$ & dide \\
\hline Sit (down) & Si $\int_{i}$ & tSet $\int i$ & tìiśí & tèés̃i & teéśíi & tSét Si & t Jit ś́ & $t \int i t \int i$ & tSetSé & d3òkó \\
\hline Climb & jí & jí & jí & jí & ji & ji & jí & ji & jí & gī \\
\hline Walk & $d \overline{3} \overline{1}$ & d3z̀े & $s \bar{\varepsilon}$ & $s \bar{\varepsilon}$ & š̀ & $s \bar{\varepsilon}$ & $s \bar{\varepsilon}$ & $\int \bar{\varepsilon}$ & sغे & $\overline{11}$ \\
\hline Enter & $\begin{array}{l}\text { sógúb } \\
\text { ára }\end{array}$ & sóso & $\Phi \varepsilon$ ve & фéve & Ф́́ve & фर́ve & фéve & $\Phi \varepsilon \phi \mathrm{e}$ & $\phi \grave{\varepsilon े}$ & wolé \\
\hline Arrive & bo & radze & wódí & po & dí & úvadi & vádí & kpò & po & dé \\
\hline Show & gbàga & gbàga & gbàga & gbàga & gbàga & gbàga & gbàga & gbàga & gbàga & fikīā \\
\hline Want & bi & $f f^{\prime}$ & f́́ & bè & f'́ & bé & t Só & tSó & tsó & wá \\
\hline Reply & dáhũ & dáhū & desì & dágusi & dáīū & dáhū & dz马úts & - & góKi $\bar{\varepsilon}$ & fèsì \\
\hline $\begin{array}{l}\text { Ask } \\
\text { (question) }\end{array}$ & bìdì & bidir દે & dírì & birè & birè & (wúmà) & $\begin{array}{l}\text { (wúù } \\
\text { má) }\end{array}$ & birè & bìdè & bèrè \\
\hline
\end{tabular}




\begin{tabular}{|c|c|c|c|c|c|c|c|c|c|c|}
\hline Fight & kpídzi & pedze & wर́dzว & d3à & dzà & dzà & lpidzi & dzí & kpidzi & d3à \\
\hline Call & só & sóni & $\Phi \dot{e}$ & $\Phi \dot{e}$ & $\phi \dot{~}$ & $\phi \dot{e}$ & $\Phi \dot{e}$ & $\phi \dot{e}$ & $\phi \dot{e}$ & kpè \\
\hline Fear & sóho & sohó & rohó & rohó & rohó & rohó & rohó & rohó & rohó & bèrù \\
\hline $\begin{array}{l}\text { Take off } \\
\text { (Clothes) }\end{array}$ & hó & hó & ho & kó & kó & ko & kó & & hó & $\begin{array}{l}\text { bó } \\
\text { (Èwù) }\end{array}$ \\
\hline Push & fi & tì & tì & tì & tì & tìtú & tì & tìtú & tì & tì \\
\hline $\begin{array}{l}\text { Wring } \\
\text { (clothes) }\end{array}$ & fó & fó & fó & fóróò & fó & fóròó & fóròó & fóròó & fóròó & $\begin{array}{l}\text { fũ } \\
\left(\mathrm{a} \int \mathrm{s}\right)\end{array}$ \\
\hline Pour & $\int u$ & Su & sì & sì & sì & sì & sì & sì & sì & dà \\
\hline Finish & tấ & tấ & tấ & tấ & tấ & tấ & tấ & tấ & tấ & tấ \\
\hline Catch & hû́ & hû́ & hû́ & hû́ & hû́ & Kî́ & Kî́́ & hú́ & Kû́ & mú \\
\hline Forget & bĩ & mábi & $\begin{array}{l}\text { (gbàg } \\
\text { bé) }\end{array}$ & (lávé) & wi & wi & wi & wi & $\overline{\text { wi }}$ & gbàgbé \\
\hline Weep & (gogò) & dze & wò & wò & wò & wò & wò & wò & wò & sokû́ \\
\hline Say & (kếró) & dž́îh & gb & ᄁ)m̄gb' & dò & nm̄̄gbé & dzé & dze & dze & so \\
\hline Like & f́́rấ & f $\tilde{\varepsilon}$ & $f \varepsilon^{\prime}$ & féfliie & f́́ch $\bar{\varepsilon}$ & fع غ̀rá & jé & fर́ćrà & f́́rã & f́́rẫ \\
\hline Lose & (ts j̄lli) & ráwu & ráwu & rawù & fōàwu & údíorà & ráwu & hólò & ráwu & sonù \\
\hline Get & dí & dí & ríфe & ríфe & ríфe & ríфe & ríфe & ríфe & ríфe & rígbà \\
\hline Run & sùkò & se & Sí & Si & $\phi \mathbf{i}$ & $\phi \mathbf{i}$ & Sí & Sí & Si & Sáré \\
\hline $\begin{array}{l}\text { Turn } \\
\text { around }\end{array}$ & jíkpo & sàkpo & jābúsi & Łpòji & kpòji & kpòjì & soso & kpòjì & kpòjì & kpòjì \\
\hline Follow & tise & tSit Se & wùr $\varepsilon$ & vèrर́ & vòrर́ & odó & ódò & ródò & òdó & tèlé \\
\hline Mould & mó & mò & ma & má & má & ma & má & ma & má & mo \\
\hline Burn & t $\int \tilde{a}$ & t $\int \tilde{a}$ & tû́ & tû́ & tû́ & dò & dò & dû́ & dò & d3ó \\
\hline Bury & lú & lú & lù & lù & lù & d3ù̀ & d3ù & d3ù̀ & lù & $\mathrm{Si}$ \\
\hline Hoe & kó & hò & Đgà & Đgà & நgà & (roko) & நgà & நgà & Đgà & roko \\
\hline Surpass & kèwè & SD̄vé & kèvé & kèvé & kèvé & kèvé & ts ittsivé & kèvé & kèvé & tajo \\
\hline
\end{tabular}

Note: Non-cognate items in Akokoid are put in brackets.

\subsection{DISCUSSION ON THE COMPARATIVE WORDLIST/FINDINGS}

When one looks at the variations exhibited above, one wonders how, in spite of these variations, there is intelligibility among the speakers of the nine speech forms under investigation. The point is that 'intelligibility' is a matter of degree, ranging from total intelligibility to total unintelligibility. Our findings reveal that:

(i) Arigidi and Erushu

(ii) Afa, Udo, Oge and Aje

(iii) Oyin, Igashi and Uro

One to three above are organised or arranged in a dialect continuum, in which a chain of adjacent varieties are mutually intelligible, but pairs taken from opposite ends of the chain are not. Thus: Arigidi and Erushu are mutually intelligible. The same thing applies to Afa, Udo, Oge, and Aje. Oyin, Igashi and Uro are also mutually intelligible. Intelligibility is also mutual between Udo, a member of group (ii) above and Oyin, a member of group (iii) above. The reason, according to the Oloyin of Oyin, Oba L.O. Bamisile, is that Udo and Oyin were together until 1922, when Udo migrated to Oke Agbe to join Afa, Oge and Aje to form Oke Agbe. The story is similar for Igashi and Uro which were together until 1955 when Uro left to join Daja, Ojo, Efifa, Iludotun, Oso, Ora and Esuku to form 'Ajowa community'. 


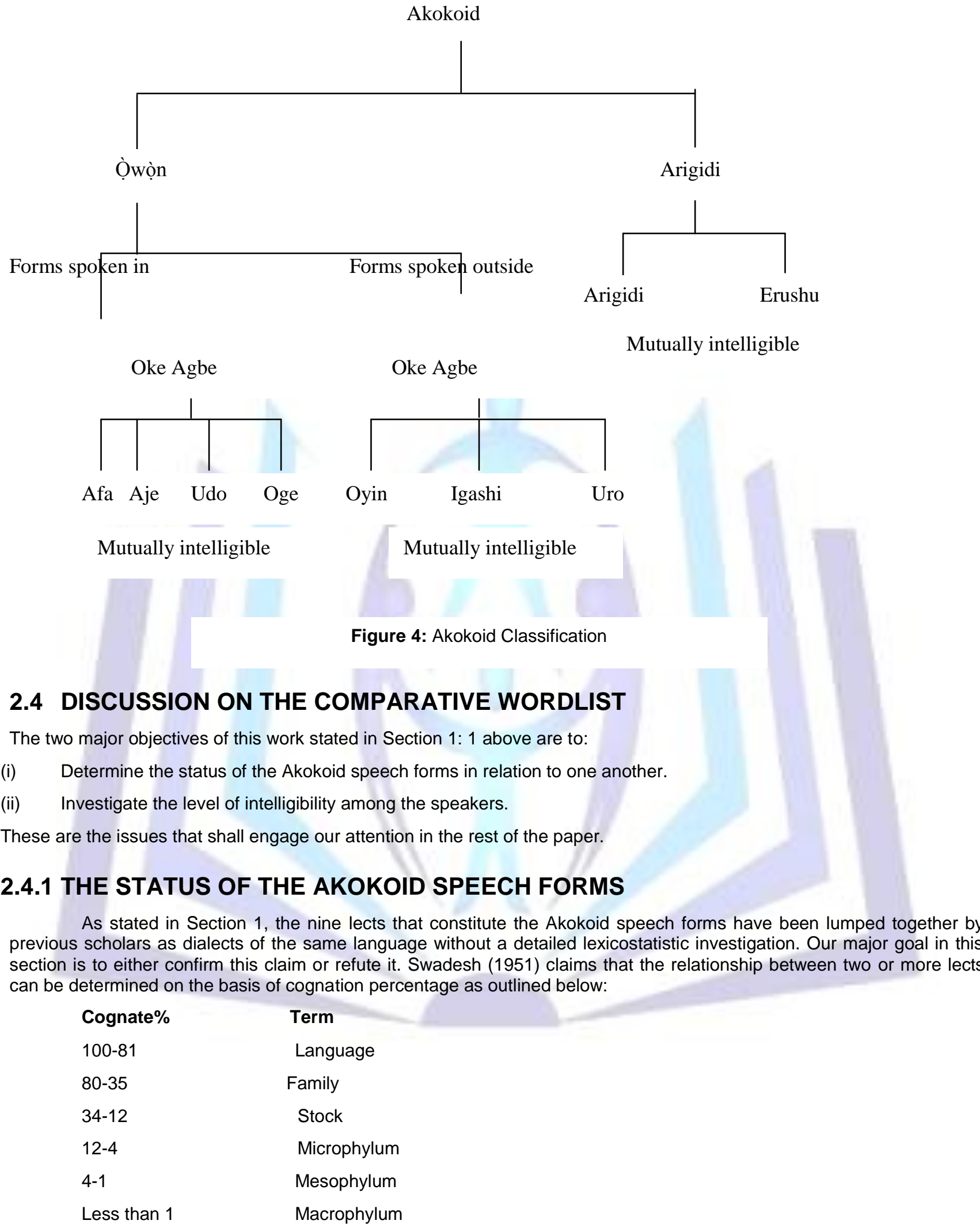

Our lexicostatistic count on the basis of the 200 lexical items above, shows that 152 items, which constitute $76 \%$, are virtual cognates. Thus by Swadesh's standard, the nine speech forms belong to the same family. We refer to this family as Akokoid. A deeper look, produces the following breakdown:

- Arigidi and Erushu average $88.5 \%$ between themselves. This figure qualifies them as dialects of the same language, referred to as Arigidi. 
- $\quad$ Afa, Udo, Oge, Aje (Ese), Oyin, Igashi and Uro average 81\% among themselves. This qualifies them as dialects of the same language, which we refer to as Ộọ̀n. The term is particularly preferred in this work because it means 'tongue' in the seven speech forms, even though its previous use was restricted to Afa, Udo, Oge and Aje. The overall title used is 'Akokoid'. It has a general application by virtue of the fact that all the speech forms are located in Akoko.

- $\quad$ Arigidi and Owọ $n$ average $76 \%$ between themselves; this established them as two different but related languages. They are jointly referred to as "Akokoid" in this work, as shown below:

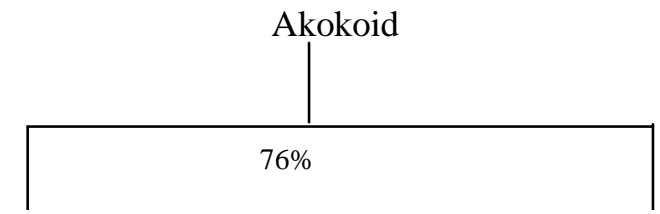

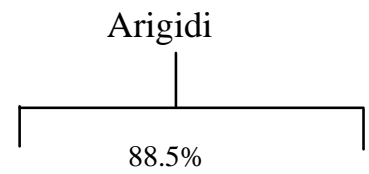

Arigidi

Erushu

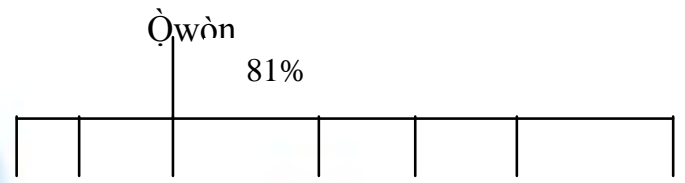

Afa Oge Aje (Ese) Udo Oyin Igashi
Uro

Figure 5: Akokoid according to cognatıon percentages

\subsubsection{MUTUAL INTELLIGIBILITY}

Ideally, the distinction between language and dialect is based on the notion of mutual intelligibility. Dialects of the same language should be mutually intelligible, while different languages should not be. Mutual intelligibility is a reflection of the linguisitic similarity between the different varieties of speech. From our data above, the issue of linguistic similarity is conspicuous. For instance, the $88.5 \%$ cognation between Arigidi and Erushu implies a considerable similary between the two lects. The same thing applies to the Owọ̀n lects, which share $81 \%$ cognation among themselves. When we compare this with the $76 \%$ between the two groups - Arigidi and Owọn, it would be reasonable to draw the conclusion that the level of intelligility within each group is very high. Let us extract some data to show this.

TABLE 2: AKOKOID/EDOID

\begin{tabular}{|c|c|c|}
\hline Arigidi & Owon & Gloss \\
\hline egírí & igírí & Head \\
\hline ód3ù & ídzù & Eye \\
\hline oto & útó & Ear \\
\hline$\varepsilon \dot{p} 0$ & ípo & Breast \\
\hline ogo & igo & Belly \\
\hline ówo & úwó & Hand \\
\hline ùho & ùhò & Leg \\
\hline èdze & $i d 3 i$ & Body \\
\hline uto & ìtó & Urine \\
\hline$\varepsilon \mathrm{m} \varepsilon$ & ímí & Feaces \\
\hline ed3i & ùd3i & Water \\
\hline umu & $\operatorname{Imu}$ & Palm Wine \\
\hline óhũ & ínru & Okra \\
\hline อ́h & ũyro & Tree \\
\hline
\end{tabular}

The Edoid items are extracted from Elugbe (1989)

These are just few examples of total cognates within each group among several other ones. Apart from this, there are many others which are examples of partial cognates. Our findings reveal that the following are intelligible:

(i) Arigidi and Erushu

(ii) Afa, Udo, Oge and Aje

(iii) Oyin, Igashi and Uro

I to III above are organised or arranged in dialect continuum, in which a chain of adjacent varieties are mutually intelligible, but pairs taken from opposite ends of the chain are not. Thus Arigidi and Erushu are mutually intelligible. The same thing applies to Afa, Udo, Oge and Aje. Oyin, Igashi and Uro are also mutually intelligible. Intelligibility is also 
mutual between Udo, a member of group (ii) above and Oyin, a member of group (iii) above the reason, according to the Oloyin of Oyin, Oba L.O. Bamisile, is that Udo and Oyin were together until 1922, when Udo migrated to Oke Agbe to join Afa, Oge and Aje to form the Oke Agbe settlement. In fact, it was said that the Oloyin had agreed to join them earlier, but as he was preparing to go with them, he was warned by his drummer through the talking drum not to follow them. Likewise, Igashi and Uro, were together until 1955, when Uro left to join Daja, Ojo, Efifa, Iludọtun, Oso, Ora and Esuku to form the 'Ajo wa Community.' This account was given by the traditional ruler of Uro Ajo wa, Oba Timothy Sunday Ipinmoroti, who was one of our informants. Thus, there is mutual intelligibility between Uro and Igashi to date because they still interact closely. However, the case is different when one compares Arigidi or Erushu with Afa or Aje (which are members of group (ii) above) or when one compares Arigidi with Oyin (a member of group (ii) above. There is a partial intelligibility. However, it is pertinent to note that intelligibility exists among these speech forms in varying degrees because the overall distance is not considerable and a lot of intermarriages still go on among them. The point we are emphasizing here is that the similarity that exists among the speech forms is not produced by accident. Obviously, they descended from the same proto-form; therefore; they are 'sisters'. So, we postulate a common source for them, in the name of 'Akokoid'. This is summarised in the tree diagram below;

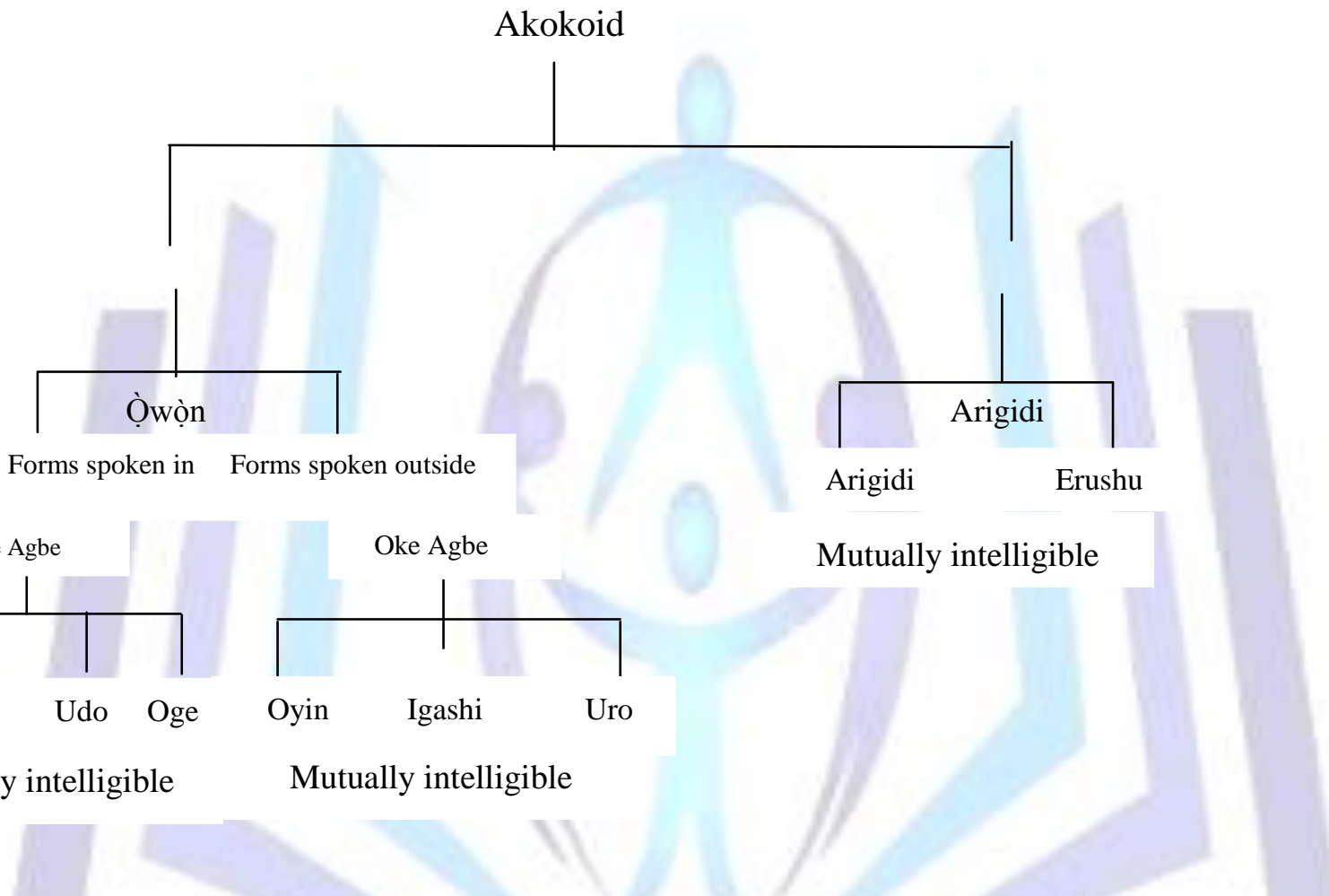

Having discussed the two key issues we highlighted as our objectives, what is now left for us is to discuss how variables like age, gender, location and migration patterns have affected our result.

\section{AGE}

The ages of individual informants have a serious effect on their mastery of their speech forms. Out of the 34 informants used in this research, only 7 were young. The remaining 27 were old. The major problem we had with these young informants was the level of mastery of their speech forms. In fact, during our sessions with them, several gaps were created, at intervals when asked: "What do you call this item in your speech form?" They would respond: "We don't know". Then we would have to resort to the older informants who would quickly supply the data. Several times, the data our young informants supplied were actually Yoruba words and, in an attempt to validate their answer, we would turn to the elders who would tell us the answer in their speech forms. This is a confirmation that these young ones were deficient in the mastery of their speech forms. This aligns with Oyetade's (2007) observation that "ability in Akoko languages among the young ones is gradually dwindling, such that they are not as proficient in their speech forms as the adult speakers." In fact, during our field trips, parents complained bitterly that their children could no longer speak their languages. This is a serious indication that the process of language shift among the youth is ongoing.

\section{SEX}

Research in Western nations affirms that women's speech is considered to be more self-conscious and classconscious than men's speech, such that data collected from them are not as reliable as those from men because they pretend to be sophisticated and artificial in their utterances. Conversely, men's speech is 'purer' and more original because they are rather 'conservative' (see Chambers and Trudgill 1998 and Francis 1983). This seems to be the case with our female informants in Akokoid. In fact, it was obvious that the men were more competent than their female 
counterparts. We had to turn to the male informants several times because the female informants were not able to satisfactorily supply our data. That is why only 5 informants were selected from the female, whereas 29 were males. Oyetade (2007) has reported that the male informants have a higher proficiency in their speech forms than their female counterparts.

\section{LOCATIONS}

Out of our 34 informants, 32 lived in their different Akoko villages, while only 2 lived in Ibadan as at the time of data collection. Our experience confirms that these mobile informants are not as proficient as their non-mobile (stationary) counterparts in their mastery of their speech forms. This is natural and logical. The fact that they have been away from their root for years has detached them somehow from their mother tongues. They were now more comfortable with Yoruba and English which their dwelling in city has better exposed them to Traditional dialectologists believe that the use of nonmobile informants guarantees that their speech is characteristic of the region in which they live, free from any external influence.

\section{MIGRATION}

According to the traditional rulers of Uro and Oyin, all their ancestors were together in Ile-Ife at the initial stage. But as a result of threats of war, they migrated to Benin. They later had to leave Benin, and migrated to a hilly settlement near their present locations. In 1922, Revd. Lennan, an Anglican priest, encouraged them to leave their hilly settlement because they were isolated. So, it was in 1922 that the first wave of migration took place. More waves of migration followed later, as we have discussed earlier. These waves of migrations have occasioned the differences we see in our data. A careful look at our data also shows that many lexical items are cognate with their Yoruba equivalents. For instance, let us look at the forms for king, cow, cat, cassava, toad, tortoise, crab, bee and snail, and so on. In fact, Fadoro (2013) forthcoming claims that Akokoid is 36\% cognate with Yoruba. Also the migration to Benin has left its mark on the speech forms. An extract from Elugbe (1989) will show this.

\begin{tabular}{|l|l|l|}
\hline Gloss & Akokoid & Edoid \\
\hline Cotton & Oruru & oruru \\
\hline Lizard & òkòtò & ókído \\
\hline Bird & õ̃ã & óura \\
\hline Nose & úw̃̃̃ & úw̃̃̃ \\
\hline Hawk & àpòpò & ákpukpo \\
\hline Cap & ìdū & ètù \\
\hline Year & èbō & èzō \\
\hline Kill & kpú & kpé \\
\hline Roast & tũ & tõ \\
\hline Mould & mã & mã \\
\hline Vomit & kpà & kpa \\
\hline
\end{tabular}

The Edoid items are extracted from Elugbe (1989).

A look at the above items reveals a striking similarity. It would not be out of place if one describes them as cognates. From the foregoing, we can safely assert that the migration patterns of these people have left their marks on their speech forms in terms of some of the similarities and variations that exist till today.

\subsection{CONCLUSION}

In this work, an attempt has been made to examine the relationship within the Akokoid speech forms. The data used for this work show that the speech forms are closely related with minor differences at the phonological and lexical levels. In most cases, the items are 100\% cognate in terms of consonants, vowels and tones; in some cases, there are minute differences. The similarity of these speech forms in the data establishes a common ancestry for the speech forms. Apart from that, it proves that truly language is not monolithic and that variation in language is a norm rather than an exception. Through the lexicostatistic analysis, we are able to conclude that the nine speech forms are classifiable into two different but related languages, which are Arigidi and Owọ̀n. This classification is also supported by mutual intelligibility. Finally, the nine speech forms constitue a dialect continuum.

\section{REFERENCES}

[1] Akinkugbe, O.O. 1978. A comparative phonology of Yorùbá dialects, Itsekiri and Igala. Ph.D thesis, University of Ibadan. 
[2] Akinyemi, O.V. 2002. A study of the internal relationship within Northern Akokoid. Unpublished B.A., Project, University of lbadan.

[3] Bright, W. \& Rameajaan, A.K. 1964. Sociolinguistic variation and language. Proceedings of the $9^{\text {th }}$ International Congress of Linguistics. H. Lint (Ed.). Cambridge, Massechusetts, The Hague: Monton. 1107 - 12.

[4] Bright, William (ed.) 1966. Sociolinguistics. The Hague: Mouton.

[5] Brook, G.L. 1973. Varieties of English. London: Macmillan.

[6] Capo, H. B. C. 1989. Defoid, The Niger-Congo Languages. J. Bendor-Samuel (Ed.) New York and London: University Press of America. Pp. 275 - 90.

[7] Chambers, J.K. \& P. Trudgil 1998. Dialectology. Cambridge: Cambridge University Press.

[8] Dada, S. A. 2006. Erushu-Yorùbá endoglossic bilingualism and language alternation in Akoko, South-western Nigeria. Unpublished Ph.D. thesis, University of Ibadan.

[9] Elugbe, B.O. 1989. Comparative Edoiod: phonology and lexicon. Port-Harcourt: University of port-Harcourt Press.

[10] Fadoro J.O. 2010. Phonological and Lexical Variations in Akokoid. Unpublished Ph.D thesis, University of Ibadan, Ibadan.

[11] Fadoro 2013. (Forthcoming). The status of Akokoid in relation to the Yoruba language.

[12] Francis, W.N. 1983. Dialectology: an introdeuctin to language. Harcourt Brace Jonavanovish.

[13] Fromkin, V. \& R. Rodman 1993. An introduction to language. (5 $5^{\text {th }}$ edition): Harcourt Brace Jovanovich.

[14] Hoffman Carl 1974. The languages of Nigeria by language family. An index of Nigerian languages, H.J. BendorSamuel and R. Stanford (Eds.) Ghana: Summer Institute of Linguistics, pp. $169-190$.

[15] Hudson, J. A. 1996. Sociolinguistics. Cambridge: Cambridge University Press.

[16] Oyètádé S.O. 2007. Language endangerment in Nigeria: perspectives on Akoko languages of the Southwest. Dorian. N.C., (ed.) Small languages and small language communities. Int'l. Journal of Sociology of Language. Pp. $169-184$.

[17] Swadesh, M. 1972. The origin and diversification of language. London: Routledge \& Kegan Paul. 